山्山FANÇAISE

$>\mathrm{DE}$

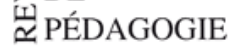

Revue française de pédagogie

Recherches en éducation

197 | 2016

Les multiples facettes de la créativité dans

l'apprentissage

\title{
Une recherche écologique pour développer la flexibilité et les relations sociales dans la créativité artistique
}

Ecological research to develop flexibility and social interactions in artistic creativity

Marion Botella et Todd Lubart

\section{(2) OpenEdition}

Journals

Édition électronique

URL : https://journals.openedition.org/rfp/5137

DOI : $10.4000 /$ rfp.5137

ISSN : 2105-2913

Éditeur

ENS Éditions

Édition imprimée

Date de publication : 31 décembre 2016

Pagination : 13-22

ISSN : 0556-7807

Référence électronique

Marion Botella et Todd Lubart, « Une recherche écologique pour développer la flexibilité et les relations sociales dans la créativité artistique », Revue française de pédagogie [En ligne], 197 | 2016, mis en ligne le 31 décembre 2019, consulté le 06 janvier 2022. URL : http://journals.openedition.org/rfp/5137 ;

DOI : https://doi.org/10.4000/rfp.5137 


\title{
Une recherche écologique pour développer la flexibilité et les relations sociales dans la créativité artistique
}

\author{
Marion Botella \\ Todd Lubart
}

\begin{abstract}
Cet article présente une étude concernant le développement de la flexibilité cognitive et des relations interpersonnelles dans le cadre de la résolution d'une tâche de création artistique. Des exercices ont été proposés à des étudiants en arts plastiques afin de les aider à développer leur créativité. Grâce à des comparaisons pré-/post-test, cette étude a permis de mettre en évidence la possibilité, par des exercices simples, de modifier des aspects spécifiques du processus créatif. Cette recherche illustre des pistes de création pédagogique afin de favoriser la démarche créative puisque nous avons montré que, selon le profil des étudiants, certains ont besoin de renforcer leur flexibilité et d'autres le lien social. II s'agit d'une pédagogie différenciée pour la créativité où l'apprentissage de la créativité serait différencié selon le profil des apprenants.
\end{abstract}

Mots-clés (TESE) : créativité, activités créatrices, pédagogie différenciée, exercice

\section{Introduction}

La créativité est la capacité à produire des idées qui soient à la fois originales et adaptées (Anderson, De Dreu \& Nijstad, 2004; Lautrey \& Lubart 1998; Lubart, 1994 ; Lubart, Mouchiroud, Tordjman et al., 2015 ; Runco \& Jaeger, 2012; Sternberg \& Lubart, 1999). Elle est généralement associée au domaine artistique même si elle intervient dans de nombreux autres domaines tels que les sciences, le design, la musique, l'écriture, etc. L'art peut être considéré comme un domaine prototypique de la créativité.

Les travaux de grands artistes tels que MichelAnge, Léonard de Vinci, Van Gogh ou Picasso ont longtemps été objets d'études psychologiques (Piirto, 1992). Dans les réflexions théoriques sur le fondement de l'imagination artistique, de nombreuses conceptions ont été proposées (Ehrenzweig, 1967). L'artiste ne doit pas simplement être plus imaginatif ou inventif que les autres pour produire un travail. Il doit créer un 
objet actif qui interagit psychologiquement avec le spectateur. Les chercheurs ont alors tenté d'identifier les facteurs jouant un rôle dans la créativité artistique.

Par exemple, Feist (1998), dans une méta-analyse sur les traits de personnalité liés à la créativité scientifique et artistique, montre que les artistes sont plus ouverts aux expériences, à la fantaisie et à l'imagination. Ils sont aussi plus impulsifs et moins consciencieux que des populations témoins, non artistes. Sur un plan émotionnel, ils se révèlent plus anxieux, labiles et avec une forte sensibilité aux émotions. Les artistes sont des personnes entraînantes et ambitieuses. Ils remettent souvent les normes en cause et sont anticonformistes. Se rebeller contre les normes établies par la société est un trait fort chez les artistes (Feist, 1999). Leur anticonformisme leur permet d'interroger, de contester les règles et de repousser les limites.

Plus récemment, des entretiens avec des artistes professionnels, c'est-à-dire des artistes ayant déjà exposé au niveau national et international vivant de leur activité artistique, ont mis en évidence l'importance de deux facteurs : la flexibilité et les relations interpersonnelles (Botella, Glaveanu, Zenasni et al., 2013). Ces deux facteurs, bien connus des chercheurs en créativité, seront présentés dans les parties suivantes, puis nous décrirons les résultats d'une étude empirique dans laquelle nous avons proposé des exercices à des étudiants en art pour améliorer l'un ou l'autre des facteurs.

\section{Flexibilité}

Adhérer à une idée sans envisager les autres possibilités inhibe la créativité (Levy \& Langer, 1999). La souplesse de pensée est donc très importante pour l'individu créatif. La flexibilité est la capacité à analyser un problème sous plusieurs angles (Scott, 1999; Thurston \& Runco, 1999). Par exemple, Ritter, Damian, Simonton et alii (2012) ont manipulé quatre conditions expérimentales grâce à la réalité virtuelle : a) les étudiants étaient immergés dans la condition virtuelle «inhabituelle et inattendue» qui violait les règles de la physique (objet rétrécissant lorsque l'avatar s'approche, une bouteille qui s'envole au lieu de tomber...); b) les étudiants étaient immergés dans la condition virtuelle «normale» présentant les mêmes situations mais en respectant les règles de la physique; $c$ ) dans la condition «vicariante et inattendue», les étudiants vision- physique sans être immergés dans la réalité virtuelle; et d) dans la condition «vicariante et normale», les étudiants visionnaient le film des évènements respectant les règles de la physique. Les auteurs ont montré que la condition «immersion virtuelle en condition inhabituelle et inattendue» augmente significativement la flexibilité par rapport aux autres conditions, soulignant ainsi le fait qu'expérimenter activement des évènements inhabituels favorise la flexibilité évaluée par la tâche des usages inhabituels où les étudiants doivent générer autant d'idées que possible en temps limité en répondant à la question "qu'est-ce qui fait du bruit?» (Guilford, 1967). Ainsi, on peut constater que la flexibilité est un facteur essentiel à la créativité (De Dreu, Bass \& Nijstad, 2007). La flexibilité n'est cependant pas qu'un effet des consignes données aux participants mais bien une caractéristique individuelle. Lubart, Mouchiroud, Tordjman et alii stipulent que la flexibilité est «l'aptitude à appréhender un seul objet, une seule idée, sous des angles différents, la sensibilité au changement ainsi que la capacité à se dégager d'une idée initiale pour explorer de nouvelles pistes » $(2015$, p. 22).

\section{Relations interpersonnelles}

La créativité impliquerait la solitude (Storr, 1989). Les artistes sont plus individualistes et introvertis que la population générale (Feist, 1999). Ce trait se manifeste principalement par un anticonformisme et la remise en question des règles. Leur système de valeurs est non conventionnel et, par là même, leur assure une indépendance de jugement (Barron, 1969; Barron \& Harrington, 1981; Gough, 1979; MacKinnon, 1965). Cet individualisme permet d'être créatif, car le créateur ne se contente pas de ce qui est mais il remet toujours en question son environnement et lui-même afin de générer des productions nouvelles et adaptées. Cette indépendance permet d'éviter les influences sociales. En conséquence, le créateur possède une grande confiance en lui et en ses capacités (Feist, 1999; Zenasni, Besancon \& Lubart, 2008). Il peut alors présenter une certaine arrogance et se sentir supérieur aux autres. Les ressources obtenues par ses productions et un environnement compétitif peuvent renforcer ce trait mais aussi l'hostilité et la confiance en soi.

Les artistes sont souvent perçus comme rebelles, insubordonnés et remettant sans cesse en cause les normes existantes (Feist, 1999). Solitaires, ils tiennent une place particulière dans le monde social. Les 
créateurs sont assez marginaux dans le sens où l'individu appartient par «attribution, réalisation, aspiration ou auto-identification à au moins deux groupes distincts » (Dogan, 1999, p. 179). L'auteur décrit trois types de personnes créatives marginales : le pionnier (celui qui repousse les frontières de sa discipline), le constructeur (celui qui explore toutes les possibilités de sa discipline) et I'hybride (qui apporte des nouveautés d'autres disciplines). La créativité peut notamment être vue comme un comportement de non-conformisme aux normes de la société et le rejet des règles établies (Cropley, 1999). Les rapports sociaux sont donc pour eux assez difficiles.

La personnalité des artistes se définit par des traits d'indépendance, d'hostilité, de distance relationnelle et de manque de chaleur. Feist (1998) met en avant les faibles scores de socialisation obtenus par les artistes. Ils semblent peu responsables et sociables. Comme ils ne se conforment pas, ils ne font pas toujours bonne impression. À cause de leur caractère radical, réservé, impulsif et froid, les artistes sont considérés comme ayant des tendances antisociales. Drevdahl et Cattell (1958) ont interrogé 153 artistes, sélectionnés pour la reconnaissance de leur travail (prix, expositions); ils ont constaté que leurs traits de personnalité diffèrent significativement de la population générale. Les résultats indiquent que les artistes sont plus matures sur le plan émotionnel, plus dominants, plus aventureux, plus radicaux, plus sophistiqués et moins cyclothymiques et suspicieux. Getzels et Csikszentmihalyi (1976) ont aussi trouvé que les artistes manquent de chaleur dans leurs rapports aux autres. Bien que plusieurs travaux aient mis en évidence le caractère solitaire de l'artiste, Csikszentmihalyi (2006) suggère que le monde social est impliqué dans la créativité. Les artistes sont effectivement en contact avec d'autres artistes et leurs œuvres, antérieurs et contemporains, des galeristes et le public.

\section{Une recherche pour développer la créativité des étudiants en art}

\section{Objectifs de la recherche}

Alors que les études sur la flexibilité cognitive soulignent clairement son implication dans la créativité, les études sur les caractéristiques des artistes sont en contradiction avec les propos tenus par ceux-ci lors d'entretiens. Botella, Glaveanu, Zenasni et alii (2013) ont rapporté que les artistes mentionnaient l'implication de leur monde social dans leur processus créatif (incluant l'environnement personnel et professionnel composé des amis, du partenaire, de la famille, des proches, des assistants, des clients, des collectionneurs, des galeristes, etc.). Par l'analyse de ces entretiens, les auteurs ont montré que l'entourage personnel et professionnel est particulièrement important chez les femmes, chez les artistes pluridisciplinaires qui cumulent plusieurs médiums, et également chez les artistes les plus expérimentés (plus de 20 ans d'expérience). Les interactions avec le monde social seraient donc essentielles à la créativité artistique mais également à d'autres domaines créatifs (Glaveanu, Lubart, Bonnardel et al., 2013). Ainsi, bien que les recherches sur les relations interpersonnelles des artistes aient montré qu'ils étaient plutôt solitaires, individualistes et froids, les artistes ont un discours qui souligne l'importance de leur monde social dans leur créativité. II semblerait donc que, bien que les artistes aient des traits de personnalité peu favorables à l'établissement de bonnes relations interpersonnelles, ces relations soient pour autant nécessaires à leur créativité. Sur la base de ces propos, nous avons fait le choix de considérer que les relations interpersonnelles méritaient d'être améliorées à la fois concernant l'entourage professionnel et l'entourage personnel.

La présente étude porte principalement sur ces deux facteurs que sont la flexibilité cognitive et les relations interpersonnelles dans le domaine artistique'. L'objectif est de proposer des exercices à des étudiants en art afin de les aider à développer leur créativité sur l'un de ces deux facteurs et d'examiner l'effet de ces exercices sur le déroulement de leur processus créatif. Puisque la population des étudiants en art est composée majoritairement de femmes, que les étudiants sont formés à plusieurs médiums pendant leur cursus et que la formation universitaire est un temps propice pour développer des relations à la fois personnelles et professionnelles, les exercices de relations interpersonnelles pourront apporter des éclaircissements quant aux résultats contradictoires de la littérature scientifique. Cette étude propose également une recherche qui s'intègre dans le contexte d'un cours déjà existant (les étudiants ne sont donc pas sollicités pour participer à une étude expérimentale ayant lieu en laboratoire). Cet objectif permet de renforcer la validité écologique de ces résultats.

1 Cette étude s'inscrit dans le projet de recherche CREAPRO ANR-08-CREA-038-01 «Étude empirique du processus créatif dans différents domaines » financé par l'Agence Nationale de Recherche. 
Hoc et Darses (2008) précisent que l'approche écologique présente plusieurs problèmes tels que la variabilité individuelle et la taille de l'échantillon testé. De plus, il n'est pas possible de contrôler les variables influençant une situation naturelle. Se pose alors la question de la légitimité à comparer les sujets entre eux (Lewedag, Oller \& Lynch, 1994; Lewis \& Gregory, 1987), de la représentativité des participants et de la méthode à employer (Hoc \& Darses, 2008). En revanche, l'approche écologique permet l'observation et l'évaluation du phénomène étudié dans l'environnement naturel où il se manifeste. Leclerc (2005) souligne que les observations directes du processus créatif permettent : a) de valoriser la validité écologique des résultats; b) d'observer le processus dans son environnement naturel; c) de travailler sur un temps plus long que ce que permettent les études en laboratoire; $d$ ) de compléter les interprétations formulées par d'autres approches (des entretiens ou des verbalisations par exemple); e) d'examiner les facteurs environnementaux, sociaux, motivationnels entourant le phénomène étudié; et f) de découvrir d'autres facteurs potentiellement associés au processus créatif.

\section{Méthode}

\section{Participants}

Vingt-cinq étudiants en arts plastiques de l'université Rennes 2 ont participé à cette étude dans le cadre de leurs cours de gravure. L'échantillon est majoritairement composé de femmes (69\%). Dans un premier temps, les capacités de pensée flexible et de relations sociales des étudiants en art ont été évaluées. Ensuite, nous avons regardé laquelle de ces deux capacités était la plus faible pour répartir les étudiants en deux groupes: s'ils avaient plus de difficultés avec la pensée flexible, ils étaient assignés au groupe "Flexibilité» ( $n=11)$ tandis que s'ils avaient plus de difficultés relationnelles, ils étaient assignés au groupe «Social» ( $n=14)$. Dans un second temps, chaque groupe s'est vu proposer deux exercices dans le domaine où il avait le plus de difficultés. Ainsi le groupe Flexibilité a eu des exercices permettant de développer la pensée flexible tandis que les étudiants du groupe Social se sont vu attribuer des exercices où ils étaient en interactions avec d'autres étudiants de la classe. Tous les étudiants ont complété tous les questionnaires qui leur ont été présentés.

\section{Matériel}

\section{Questionnaires de flexibilité et relations interper-} sonnelles (pré- et post-test)

Pour évaluer la pensée flexible, une tâche de morphing a été utilisée avec plusieurs séries d'images qui évoluent progressivement. Il s'agit d'une épreuve utilisée classiquement pour évaluer la flexibilité. Dans cette tâche, un stimulus est présenté et se transforme au cours des essais en un autre stimulus. Par exemple, le stimulus de départ est un chat puis l'image est peu à peu déformée avec une autre image pour qu'à la fin, on distingue très nettement un papillon. Dans cette tâche, on mesure combien de déformations sont nécessaires aux étudiants pour qu'ils voient le papillon. La flexibilité correspond alors à la capacité de détection précoce du changement catégoriel.

Pour évaluer les relations interpersonnelles, un questionnaire de soutien social a été adapté à partir du questionnaire de Pinneau (1976). Il s'agit d'un questionnaire à double entrée croisant quatre types de relations interpersonnelles avec trois acteurs sociaux. Ce questionnaire a été choisi car il est rapide à remplir (12 items seulement au total) et permet cependant de calculer huit scores: I'interaction (temps que les autres ont pris pour aider à rendre le travail plus facile), la communication (facilité de parler avec les autres), l'aide (aide apportée par les autres quand les choses deviennent difficiles) et l'écoute (disposition des autres à écouter les problèmes personnels); ainsi que des scores spécifiques aux acteurs sociaux (le professeur, les camarades, et la famille/les amis). Ainsi, le score d'interaction correspond à la somme des trois catégories d'acteurs sociaux. Il en est de même pour les scores de communication, aide et écoute. Pour calculer le score lié au professeur, on fait alors la somme des réponses à l'interaction, la communication, l'aide et l'écoute pour cet acteur social. Le même calcul s'applique aux camarades et à la famille/aux amis.

\section{Carnets de bord}

Parallèlement à leurs cours de gravure, les étudiants complétaient un carnet de bord sur leurs activités créatives (étapes du processus) et sur les facteurs impliqués (pour plus de détails sur cet outil, voir Botella, Nelson \& Zenasni, 2017). Ce carnet de bord a été construit sur la base de précédents travaux (Botella, Glaveanu, Zenasni et al., 2013 ; Glaveanu, Lubart, Bonnardel et al., 2013). Ainsi, neuf activités créatives : expérimenter, accumuler (documenter, collecter), répéter, 
transformer, détruire, lier, marquer, finaliser, et attendre (regarder, ne rien faire); et neuf facteurs ont été retenus dans le carnet de bord : logique (raisonnement, planification), intuition, rêverie, volonté (persévérance, effort, discipline), dessaisissement (anarchie, déplacement, surprise, deuil), ouverture (dialogue, accueil, échange, politique), joie (excitation, enthousiasme, plaisir), sans état d'âme, tristesse (fatigue, déception, frustration). Le codage est binaire : les étudiants devaient cocher s'ils avaient effectué ou non l'activité (ou engagé le facteur) à chaque séance.

\section{Questionnaires d'impact sur la démarche créative (post-test)}

En plus des questionnaires de flexibilité et de relations interpersonnelles, les étudiants ont été invités à compléter un questionnaire supplémentaire quant à l'impact des exercices sur leur démarche créative. Ils ont alors indiqué sur une échelle de Likert en 5 niveaux à quel point l'exercice suivi les a aidés ou perturbés, leur a permis de voir les problèmes sous un angle nouveau, de s'ouvrir à de nouvelles approches, de prendre des risques, de suivre leur intuition, de plus échanger avec les autres, de mieux communiquer leurs idées, ou de prendre plus confiance en eux.

\section{Procédure}

L'étude était intégrée à leur cours de gravure de sorte à ne pas perturber leur cursus et à ce que les étudiants soient dans les conditions les plus naturelles possible et, ainsi, assurer la validité écologique de cette recherche. Les étudiants ont suivi les cours pendant 12 semaines par séance de 4 heures. Les six premières séances étaient consacrées à l'exploration pragmatique de la gravure et de ses techniques. Les étudiants ont alors reçu des directives de mi-parcours individuelles. Le pré-test a été complété pendant les vacances après cette séance 6 : les étudiants ont répondu à un questionnaire en ligne (une demi-heure de temps) sur la pensée flexible et les interactions relationnelles. À la suite de ce questionnaire, les deux groupes ont été constitués pour tenter une analyse de l'importance de ces facteurs dans la fécondité d'une démarche de création artistique. Durant cette semaine de vacances, les étudiants ont également commencé à réfléchir au projet personnel de gravure qu'ils pourraient développer dans la suite des séances.

À la septième séance, les étudiants ont commencé à travailler sur leur projet personnel. Au cours des séances 8 et 9 , nous avons testé deux exercices de pensée flexible et de relations sociales. Les étudiants du groupe Flexibilité ont dû, lors du premier exercice, travailler pour leur projet en utilisant une plaque ou une planche déjà gravée avant les vacances par euxmêmes ou par quelqu'un d'autre, et s'efforcer de la faire cadrer avec leur projet personnel. Lors du second exercice, les étudiants ont été obligés de recourir à une autre technique que celle qu'ils avaient utilisée la semaine précédente; ou bien ils devaient utiliser un procédé qu'ils n'avaient jamais essayé; ou bien encore inventer un procédé à eux. En revanche, lors du premier exercice, les étudiants du groupe Social ont été invités à discuter par binôme ou par trinôme d'étudiants constitués si possible par affinités, et à travailler en suivant les directives qui ont été données par un autre pour leur projet. Autrement dit, ce n'était pas eux qui décidaient de ce qu'ils faisaient lors de cette séance mais ils faisaient ce que quelqu'un d'autre leur avait dit de faire. Lors du second exercice, les étudiants du groupe Social ont à nouveau discuté avec leur binôme pour faire un bilan de ce que chacun avait fait à partir des directives de l'autre et ce que cela avait apporté à leur projet. Puis chacun a poursuivi son projet en tenant compte (ou pas) des remarques, conseils, critiques... du partenaire, des autres camarades, du professeur.

Enfin, les trois dernières séances étaient consacrées à la réalisation et la finalisation de leur projet personnel. À la fin du semestre, les étudiants ont à nouveau été invités à remplir le questionnaire de relations sociales ${ }^{2}$. Ils ont également expliqué les effets de ces exercices sur leur démarche créative habituelle.

\section{Résultats}

\section{Pré-test et post-test}

Les deux groupes ont été comparés avec des $t$ de Student pour groupes indépendants sur leur score de flexibilité et de relations interpersonnelles en pré-test et sur leurs relations interpersonnelles et les effets des exercices en post-test (voir tableau 1). En pré-test, les résultats indiquent que les étudiants du groupe Flexibilité ont une meilleure communication avec les autres

2 Les étudiants étant partis en vacances trop rapidement à la fin du semestre, la majorité n'a pas répondu au questionnaire de pensée flexible. Ces données en post-test n'ont donc pas été exploitables. 
Tableau 1. Moyennes et écarts-types dont la différence est significative entre le groupe Flexibilité et le groupe Social

\begin{tabular}{r|c|c}
\hline & Groupe Flexibilité & Groupe Social \\
\hline $\begin{array}{r}\text { Pré-test } \\
\text { Relations interpersonnelles }\end{array}$ & \\
Communication & & $7,27(1,68)$ \\
Aide & $10,33(2,08)$ & $6,64(1,36)$ \\
Professeur & $10,00(1,73)$ & $8,55(2,46)$ \\
Famille & $12,33(2,31)$ & $8,64(2,84)$ \\
\hline Post-test & $12,33(0,58)$ & \\
Impact sur la démarche créative & & $3,73(1,19)$ \\
Échanger plus avec les autres & & $3,73(1,19)$ \\
Mieux communiquer ses idées & $2,33(1,00)$ & $2,45(0,52)$ \\
\hline
\end{tabular}

$\left(t_{(24)}=2,68, p<0,05\right)$; ils ont plus le sentiment d'être aidés par les autres $\left(t_{(24)}=3,61, p<0,01\right)$; ils ont de meilleures relations avec le professeur $\left(t_{(24)}=2,38, p<0,05\right)$ et avec leur famille que les étudiants du groupe Social $\left(t_{(24)}=2,18, p<0,05\right)$. En revanche, le groupe Flexibilité a besoin de plus d'essais pour changer son point de vue que le groupe Social $\left(t_{(24)}=2,20, p<0,05\right)$. Ces résultats confirment que les groupes sont différenciés.

Les tests $t$ de Student pour échantillons appareillés (les mêmes personnes vues à deux reprises) n'ont pas montré d'évolution significative pour les deux groupes entre le pré-test et le post-test : les deux groupes d'étudiants n'auraient donc pas progressé. Toutefois, les résultats des tests $t$ pour échantillons indépendants (des groupes constitués de personnes différentes) n'indiquent plus de différence significative entre les deux groupes pour le questionnaire de relations sociales en post-test. Ainsi, le groupe ayant reçu l'entraînement de relations sociales a "rattrapé » l'autre groupe. Quant aux effets des exercices, les résultats indiquent que les étudiants du groupe Social ont le sentiment qu'ils leur ont permis de plus échanger avec les autres $\left(t_{(24)}=2,79\right.$, $p<0,05)$, de mieux communiquer leurs idées $\left(t_{(24)}=2,80\right.$, $p<0,05)$ et de faire preuve de plus de force de travail que $d^{\prime}$ habitude $\left(t_{(24)}=2,18, p<0,05\right)$.

\section{Effets des exercices sur le processus et les facteurs}

Pour chaque groupe, nous avons également calculé le pourcentage d'étudiants ayant coché les activités et les facteurs à chaque séance. Une différence entre deux proportions a ensuite été calculée afin de savoir si elle était significative (voir figure 1 pour exemple).

Les résultats ${ }^{3}$ indiquent que les étudiants du groupe Flexibilité ont :

- plus coché l'étape d'expérimentation que les étudiants du groupe Social aux séances 8 et 11, fait preuve de plus d'ouverture à la séance 9 , de rêverie aux séances 9,10 et 12, et de tristesse à la séance 12 ;

- moins fréquemment coché l'activité de transformation et le facteur logique à la séance 9, moins finalisé et fait preuve de moins de volonté à la séance 11, et d'intuition et de joie à la séance 12 .

Ces résultats montrent que les exercices ont permis aux étudiants du groupe Flexibilité de plus expérimenter leurs idées et de développer leur ouverture d'esprit. Les exercices du groupe Social ont permis aux étudiants de plus transformer leur idée et, au final, d'être plus satisfaits de leur production.

\section{Processus créatif et profils}

De plus, nous avons examiné le processus créatif de ces deux groupes d'étudiants et indiqué quels étaient les facteurs les plus caractéristiques de chaque activité de leur processus. Pour cela, nous avons, dans un premier

3 Seules les différences après les exercices sont mentionnées. II n'y a que quelques différences avant mais celles-ci peuvent expliquer pourquoi les groupes ont été ainsi constitués. Tous les résultats sont significatifs au seuil $p<0,05$ sauf si cela est précisé. 
Tableau 2. Processus créatif de chaque groupe selon les transitions entre les étapes (à quelle activité mène le plus fréquemment une autre activité) et les profils de chaque étape (les facteurs associés à chaque activité)

\begin{tabular}{l|l|l|l|l}
\hline \multirow{2}{*}{} & \multicolumn{2}{|c|}{ Transitions } & \multicolumn{2}{c}{ Profils } \\
\cline { 2 - 5 } & Flexibilité & Social & Flexibilité & Social \\
\hline Expérimenter & & Accumuler, attendre & Intuition, ouverture & Intuition, rêverie \\
\hline Répéter & $\begin{array}{l}\text { Transformer, } \\
\text { détruire, lier }\end{array}$ & Transformer, lier & & \\
\hline Transformer & $\begin{array}{l}\text { Détruire, } \\
\text { finaliser, attendre }\end{array}$ & Accumuler & & \\
\hline Détruire & Accumuler, répéter, lier & Accumuler, lier & Tristesse & Intuition, volonté \\
\hline Lier & Accumuler, finaliser & Accumuler, finaliser & & Joie \\
\hline Marquer & Expérimenter & Attendre & Rêverie & Logique, \\
intuition, joie
\end{tabular}

Note : les cellules vides indiquent qu'aucune transition ou aucun profil caractéristique n'émerge pour cette activité.

Figure 1. Pourcentage d'étudiants ayant coché le facteur «joie » à chaque séance de gravure selon leur groupe (Flexibilité ou Social)

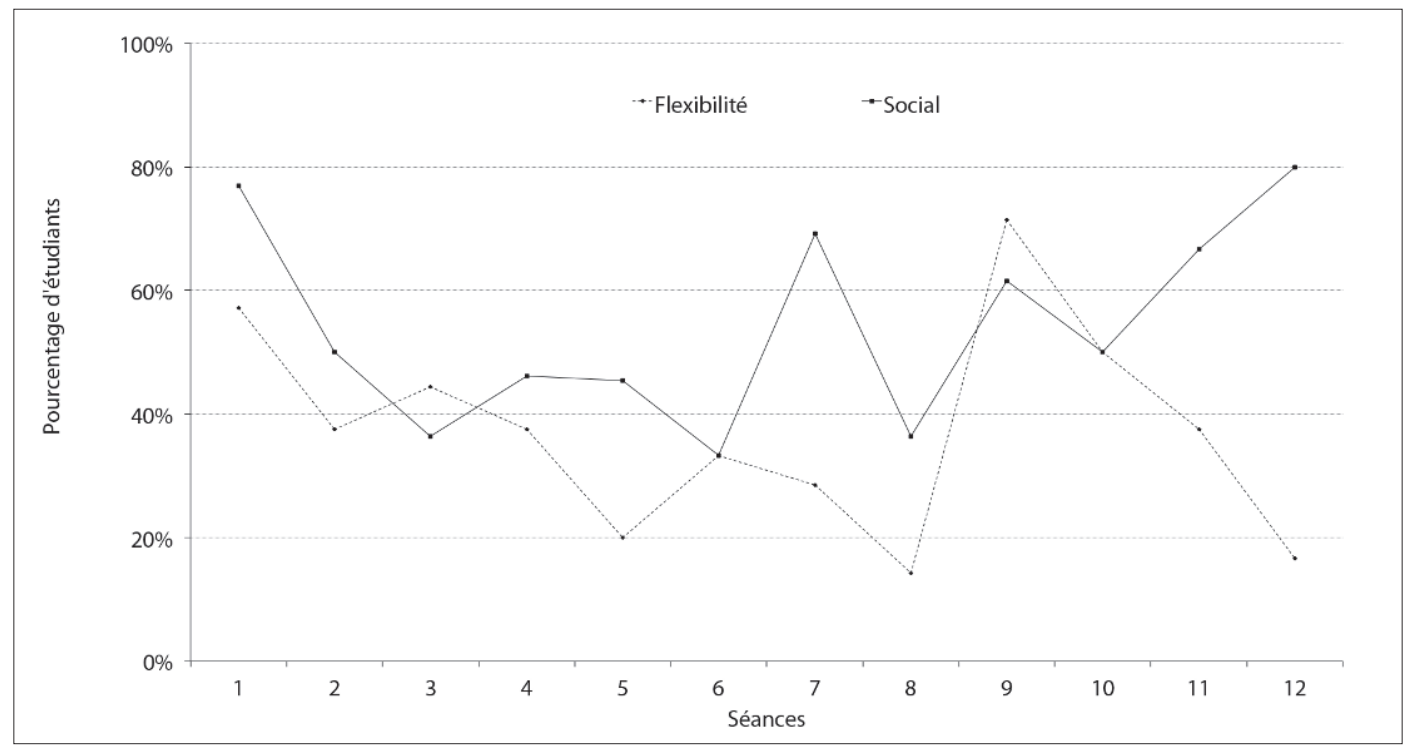

Note : $p<0,01$. 
temps, construit un tableau de transition en comptant le nombre de fois qu'une étape conduit à une autre étape. Puis, dans un second temps, nous avons mené une analyse des correspondances afin de déterminer quelles sont les transitions les plus fréquentes. De la même façon, nous avons calculé un score moyen de chaque facteur pour chaque activité du processus créatif. Puis nous avons mené une analyse de correspondances afin de savoir quel facteur est le plus caractéristique de chaque étape. Dans le tableau 2, les transitions les plus caractéristiques entre les étapes (à quelle activité mène le plus fréquemment une autre activité) et les profils de chaque étape (les facteurs associés à chaque activité) sont reportés selon le groupe. Ces résultats soulignent que le processus créatif est très différent selon le groupe, c'est-à-dire que les étudiants des deux groupes n'effectuent pas les étapes dans le même ordre et qu'une même étape ne mobilise pas les mêmes facteurs selon le groupe. Toutefois, ils permettent également de mettre en évidence des points communs entre le processus créatif des deux groupes: - l'activité d'expérimenter implique de l'intuition pour les deux groupes;

- après accumuler, les deux groupes cochent transformer et lier;

- après détruire, ils indiquent accumuler et lier;

- après lier, ils cochent accumuler et finaliser.

Cette analyse complémentaire du processus créatif de chaque groupe souligne les différences dans la démarche créative mise en place par les étudiants suite aux exercices.

\section{Discussion}

Cette étude a permis de mettre en évidence la possibilité, par des exercices simples, de modifier un peu le processus créatif des étudiants en art. D'un point de vue strictement quantitatif, les effets ne semblent pas très importants, mais dans le questionnaire final où nous leur demandions quel était l'impact de ces exercices sur leur démarche créative, les étudiants ont souligné un effet moyen de l'exercice ( $M=2,95$ sur une échelle de Likert en 5 points). Parmi les 11 étudiants du groupe Flexibilité, 6 étudiants ont commenté leur réponse :

"J'ai pu suivre au maximum mon intuition.»

«Le fait de continuer un projet qui n'était pas le mien, de ne pas me soucier de l'idée de départ, ça m'a poussé à re-questionner mon projet.»

"Cet exercice m'a permis d'avancer à un moment où
"Cela m’a permis d'avoir, au final, les résultats que je souhaitais.»

«Dans l'ensemble, je me suis laissé conduire par ce que j'avais sous les yeux.»

«J'ai pu voir comment bien manipuler la taille par rapport au rendu que je voulais.»

De même, des 14 étudiants du groupe Social, 9 ont indiqué leur sentiment sur l'impact de ces exercices sur leur démarche créative :

"C'était un stimulus intéressant, qui a en quelque sorte enrichi mon expérience et l'a rendue multiforme. En général, j'ai beaucoup aimé.»

«Parler avec une personne qui avait un recul sur mon projet m'a permis de mettre en valeur certaines choses que, moi-même, je ne voyais pas. J'ai mieux appréhendé mon travail, j'ai suivi mon intuition par rapport à ce que ma camarade m'avait dit et j'ai pris des risques.»

"Ce qui m'a aidé, c'est l'avis que peut avoir quelqu'un d'extérieur pas forcément quelqu'un qui fait de la gravure mais par exemple la famille qui donne des conseils afin d'avoir de nouvelles idées.»

"Parler avec les autres m'a permis de développer et améliorer la technique à reprendre sur une plaque déjà utilisée. Le fait de discuter avec mes camarades m'a permis de mieux construire mon projet.»

"Je pense que la contrainte qui oblige un élève à parler de son travail à un autre élève est la meilleure façon d'impliquer davantage un élève à approfondir son travail et à le rendre lisible.»

"Avant cet exercice, je ne pensais pas que mon travail allait évoluer de la sorte et le résultat fut satisfaisant !»

"L'échange avec les autres m'a donné des idées et encouragé.»

«Ce qui m'a aidé c'est le fait de partager avec mon binôme, de le sentir plus concerné par ma démarche créative.»

Les commentaires des étudiants confirment les résultats de la littérature sur l'importance de la flexibilité cognitive pour la créativité (Botella, Glaveanu, Zenasni et al., 2013; De Dreu, Baas \& Nijstad, 2007; Levy \& Langer, 1999). Ils sont également cohérents avec les propos tenus par les artistes professionnels lors d'entretiens sur leur démarche artistique qui soulignaient déjà l'influence bénéfique des autres, qu'ils appartiennent au cercle personnel ou professionnel, dans la créativité artistique (Botella, Glaveanu, Zenasni et al., 2013).

Cette étude présente plusieurs limites. Tout d'abord, nous pouvons nous demander si un semestre (soit 12 semaines de cours) est suffisant pour voir des changements dans le processus créatif des étudiants. Ensuite, la portée de cette étude est limitée par le faible nombre 
de sujets dans chaque groupe (11 étudiants dans le groupe Flexibilité et 14 dans le groupe Social). Cela limite également le pouvoir des analyses statistiques. Mais la validité écologique de l'étude a été renforcée. La validité écologique a conduit également à l'absence de groupe contrôle. En effet, dans des conditions réelles, il n'était pas possible de demander à des étudiants «de ne rien faire» pendant que d'autres avaient des exercices. L'impact de ces exercices est donc pour le moment limité, mais cette étude suggère qu'il est possible d'améliorer le processus créatif et de le faciliter.

Cette recherche illustre des pistes de création pédagogique afin de favoriser la démarche créative puisque nous avons montré que, selon le profil des étudiants, certains avaient pu améliorer leur flexibilité, et d'autres leur «lien social». Ainsi, on peut parler d'une

\section{Bibliographie}

ANDERSON N., DE DREU C. K. W. \& NIJSTAD B. A. (2004). «The routinization of innovation research: A constructively critical review of the state-of-the-science». Journal of Organizational Behavior, $\mathrm{n}^{\circ} 25$, p. 147-173.

BARRON F. (1969). Creative person and creative process. New York : Holt, Rinehart and Winston.

BARRON F. \& HARRINGTON D. M. (1981). «Creativity, intelligence, and personality». Annual Review of Psychology, $n^{\circ} 32$, p. 439-476.

BOTELLA M., GLAVEANU V., ZENASNI F., STORME M., MYSZKOWSKI N., WOLFF M. \& LUBART T. (2013). «Creative artistic activity: Factors and stages based on interviews». Learning and Individual Differences, $\mathrm{n}^{\circ} 26, \mathrm{p} .161-170$.

BOTELLA M., NELSON J. \& ZENASNI F. (2017). «It is Time to Observe the Creative Process: How to use a Creative process Report Diary (CRD) ». Journal of Creative Behavior.

CROPLEY A. J. (1999). "Definitions of Creativity». In M.A. Runco \& S. R. Pritzker (dir.), Encyclopaedia of creativity, vol. 1. New York : Academic Press, p.511-524.

CSIKSZENTMIHALYI M. (2006). La créativité : psychologie de la découverte et de l'invention. Paris : Robert Laffont.

DAVIS G. A. (1999). «Barriers to Creativity and Creative Attitudes ». In M. A. Runco \& S. R. Pritzker (dir.), Encyclopaedia of creativity, vol. 1. New York : Academic Press, p. 165-174.

DE DREU C. K. W., BAAS M. \& NIJSTAD B. A. (2007). Moodcreativity link revisited: Hedonic tone and activation level in the mood-creativity link. Paper presented at the annual conference of the Society for Personality and Social Psychology, Memphis.

DOGAN M. (1999). «Marginality». In M. A. Runco \& S. R. Pritzker (dir.), Encyclopaedia of creativity, vol. 2. New York: Academic Press, p. 179-184. pédagogie différenciée pour la créativité où l'apprentissage de la créativité serait différencié selon le profil des apprenants. Dans cet article, nous avons illustré cette pédagogie différenciée par l'exemple des étudiants en art mais cette recherche pourra être mise en application dans d'autres domaines.

Marion Botella

Université Paris Descartes, laboratoire Adaptations Travail-Individu marion.botella@parisdescartes.fr

Todd Lubart

Université Paris Descartes, laboratoire Adaptations Travail-Individu todd.lubart@parisdescartes.fr

DREVDAHL J. E., \& CATTELL R. B. (1958). «Personality and creativity in artists and writers ». Journal of clinical psychology, $\mathrm{n}^{\circ} 14(2)$, p. 107-111.

EHRENZWEIG A. (1967). The Hidden Order of Art. Londres : Paladin.

FEIST G. J. (1998). «A meta-analysis of personality in scientific and artistic creativity». Personality and Social Psychology Review, n²(4), p.290-309.

FEIST G. J. (1999). «The influence of personality on artistic and scientific creativity ». In R.J. Sternberg (dir.), Handbook of Creativity. Cambridge : Cambridge University Press, p. 273-296.

GETZELS J. W. \& CSIKSZENTMIHALYI M. (1976). The creative vision: A longitudinal study. New York : Wiley.

GLAVEANU V., LUBART T., BONNARDEL N., BOTELLA M., DE BIASI P.-M., DE SAINTE CATHERINE M., GUILLOU K., KURTAG G., MOUCHIROUD G., STORME M., WOJTCZUK A. \& ZENASNI F. (2013). "Creativity as Action: Findings from Five Creative Domains ». Frontiers in Psychology, $\mathrm{n}^{\circ} 4$.

GOUGH H. G. (1979). «A creative personality scale for the Adjective Check List». Journal of Personality and Social Psychology, n³7, p. 1398-1405.

GUILFORD J. P. (1967). The nature of human intelligence. New York : McGraw-Hill.

HOC J.-M. \& DARSES F. (2008). Quelles validités écologiques pour les recherches menées dans le GDR Psycho Ergo? Journées d'Automne, organisées les 20 et 21 novembre 2008 à Paris.

LAUTREY J. \& LUBART T. I. (1998). «Créativité». In O. Houdé, D. Kayser, O. Kœnig, J. Proust \& F. Rastier (dir.), Vocabulaire des sciences cognitives : neurosciences, psychologie, intelligence artificielle, linguistique et philosophie. Paris : PUF, p. 123-124. 
LECLERC J. (2005). Modélisation de la cognition dans le domaine des arts visuels. Mémoire de maîtrise, psychologie, Université de Montréal.

LEVY B. \& LANGER E. (1999). "Aging». In M. A. Runco \& S.R. Pritzker (dir.), Encyclopaedia of creativity, vol. 1. New York : Academic Press, p. 45-52.

LEWEDAG V., OLLER D. \& LYNCH M. (1994). «Infant's vocalization patterns across home and laboratory environment ». First Language, $n^{\circ} 14$, p. 49-65.

LEWIS C. \& GREGORY S. (1987). «Parent's talk to their infants: the importance of context». First Language, $\mathrm{n}^{\circ} 7$, 201-216.

LUBART T. I. (1994). «Creativity». In R.J. Sternberg (dir.), Thinking and problem solving. New York: Academic Press, p.289-332.

LUBART T. I., MOUCHIROUD C., TORDJMAN S. \& ZENASNI F. (2015). Psychologie de la créativité. Paris : Armand Colin, $2^{\mathrm{e} e ́ d .}$

MACKINNON D. W. (1962). «The nature and nurture of creative talent ». American Psychologist, n 17, p.484-495.

MACKINNON D. W. (1965). «Personality correlates of creativity ». In M. J. Aschner \& C. E. Bish (dir.), Productive thinking in education. Washington : National Education Association, p. 159-171.

PIIRTO J. (1992). Understanding those who create. Scottsdale : Gifted Psychology Press.
PINNEAU S. R. (1976). Effects of social support on occupational stresses and strains. Communication présentée au congrès American Psychological Association, Washington, septembre 1976.

RITTER S. M., DAMIAN R. I., SIMONTON D. K., VAN BAAREN R. B., STRICK M., DERKS J. \& DIJKSTERHUIS A. (2012). «Diversifying experiences enhance cognitive flexibility ». Journal of Experimental Social Psychology, n 48(4), p. 961-964.

RUNCO M. A. \& JAEGER G. J. (2012). «The standard definition of creativity ». Creativity research Journal, n²4(1), p. 92-96.

SCOTT T. E. (1999). «Knowledge». In M. A. Runco \& S. R. Pritzker (dir.), Encyclopaedia of creativity, vol. 2. New York: Academic Press, p. 119-129.

STERNBERG R. J. \& LUBART T. I. (1999). «The concept of creativity: Prospects and paradigms». In R.J. Sternberg (dir.), Handbook of creativity. New York : Cambridge University Press, p. 3-15.

STORR A. (1989). Solitude. New York : The Free Press.

THURSTON B. J. \& RUNCO M. A. (1999). «Flexibility». In M. A. Runco \& S. R. Pritzker (dir.), Encyclopaedia of creativity, vol. 1. New York : Academic Press, p.729-732.

ZENASNI F., BESANÇON M. \& LUBARTT. I. (2008). «Creativity and tolerance of ambiguity: An empirical study ». Journal of Creative Behavior, $\mathrm{n}^{\circ}$ 42(1), p.61-73. 